\title{
Diurnal Changes of Heart Rate and Sympatho-Vagal Activity for Temporal Patterns of Transient Ischemia
}

\author{
A Smrdel, F Jager \\ Faculty of Computer and Information Science, University of Ljubljana, Ljubljana, Slovenia
}

\begin{abstract}
Using all 86 records of the Long-Term ST Database we studied diurnal variations of ischemia and heart rate among patients exhibiting different temporal patterns of ischemia: salvo, periodic and sporadic pattern. The results show, that the incidence of ischemia increases during the morning interval. The decrease of sympathetic and vagal activity during ischemia is the most prominent for the sporadic group, while for the salvo group only minor changes were observed. The results support our hypothesis, that there are at least two distinct populations which differ according to mechanisms and temporal patterns of ischemia.
\end{abstract}

\section{Introduction}

Long term ambulatory ECG records are often used for the analysis of transient ST segment changes compatible with ischemia (ischemic episodes). Previous study [1] described three different temporal patterns of ischemia in 2hour records of the "European Society of Cardiology ST-T Database" (ESC DB) [2]: salvo pattern (episodes appear in short bursts), periodic pattern (episodes appear quasi periodically, separated with longer intervals) and sporadic pattern (episodes appear without regularity). We set a hypothesis, that different mechanisms are responsible for different temporal patterns, namely that the salvo pattern is caused due to the vasospasms (supply driven ischemia), while the sporadic pattern is caused due to the physical exertion (demand driven ischemia). The short duration of records in the ESC DB does not permit accurate division according to temporal patterns. The "Long-Term ST Database" (LTST DB) [3], which contains 24-hour annotated records, abolishes this limitation. With numerous samples of the three temporal patterns, the LTST DB allows studies of physiological mechanisms responsible for ischemia as well as studies regarding diurnal changes.

In this paper, using the LTST DB, we test the hypothesis that different physiologic mechanisms are responsible for different temporal patterns. We studied time- and frequency-domain parameters of the instantaneous heart rate (IHR) in the neighborhood of ischemic episodes for records exhibiting distinct temporal patterns (salvo, periodic, sporadic) and for different intervals of records (day, night and morning interval).

\section{Methods}

We divided each record of the LTST DB into day, night and morning interval (figure 1). For each record we identified the interval, when the patient was asleep (the night interval) by observing trends of heart rate (lower and smoother), time series of QRS complex Karhunen-Loève coefficients (sudden step changes), and noise (not as frequent). The morning interval was defined as a 90 minute interval following the night interval. The rest of the record was considered to be the day interval. Next we divided records into groups using visual examination of time series of ST segment deviation levels. We identified the salvo pattern in 6 records, s20021 (figure 2, upper), s20151, s20171, s20291, s20301 and s20311, periodic pattern in 12 records, s20041, s20111, s20121, s20131, s20181, s20261, s20271, s20411, s20511, s20611, s30671 and s30681 (figure 1), and sporadic pattern in 24 records, s20031, s20081, s20101 (figure 2, lower), s20191, s20251, s20331, s20341, s20351, s20361, s20381, s20391, s20431, s20441, s20451, s20481, s20491, s20551, s20571, s20601, s30701, s30731, s30751, s30791 and s30801. Remaining records exhibited no distinct pattern or had no ischemic episodes. We then derived uniformly resampled $(\Delta T=0.5 \mathrm{~s})$ and smoothed (3-point moving average) IHR time series. Next we derived time- and frequency-domain parameters over a few intervals in the neighborhood of ischemic episodes: 3-minute interval starting $6 \mathrm{~min}$ prior to ischemia onset $\left(B_{6-3}\right), 3$-minute interval starting 3 min prior to ischemia onset $\left(B_{3-0}\right)$, 3-minute interval starting at the ischemia onset $\left(I_{0-3}\right)$, and interval during ischemia $(I)$. From obtained measurements we derived aggregate average statistics for all groups during all intervals of records. In the frequency domain, we obtained two time series: LF - fraction of the total IHR power in the low-frequency band (from $0.04 \mathrm{~Hz}$ to $0.15 \mathrm{~Hz}$ ), and $\mathrm{HF}$ - fraction of the total IHR power in the high-frequency band (from $0.15 \mathrm{~Hz}$ to $0.4 \mathrm{~Hz}$ ). To compute 


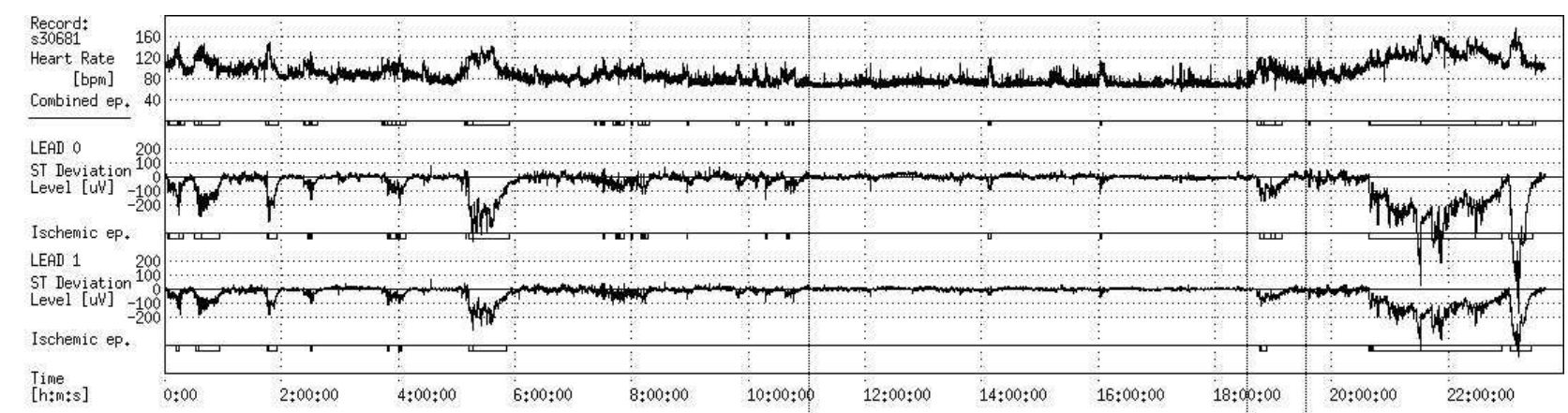

Figure 1. Time trends of the record s30681 (first two leads) from the periodic group (24 hours, recording starts at 11:35:00). Vertical lines separate day, night, morning and day interval. Legend: From top to bottom: heart rate ([bpm]); ischemic episodes from all leads, combined in the sense of logical OR function; ST segment deviation for lead $0([\mu \mathrm{V}])$; ischemic episodes for lead 0; ST segment deviation for lead $1([\mu \mathrm{V}])$; ischemic episodes for lead 1.

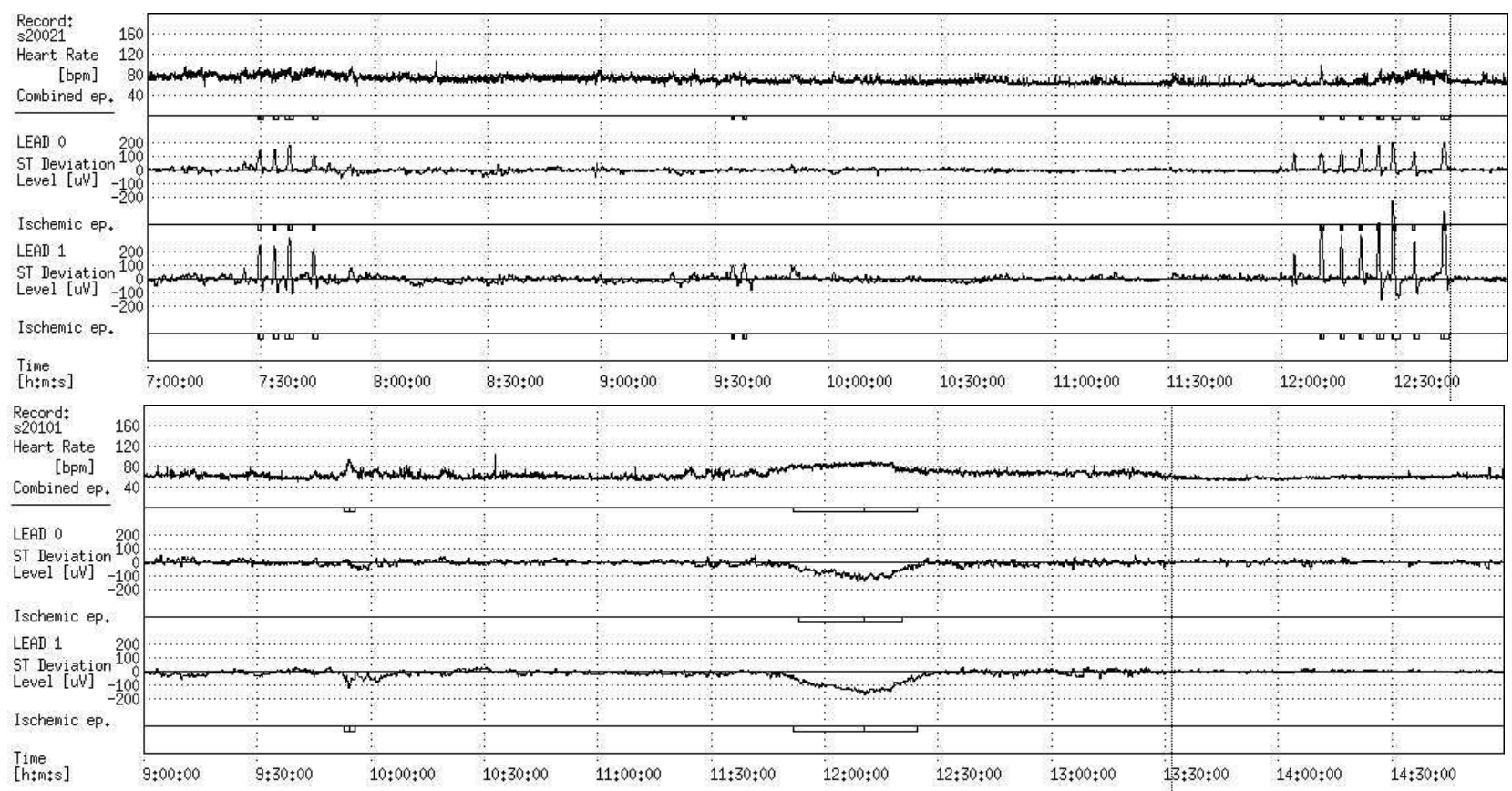

Figure 2. Time trends of the record s20021 from the salvo group (upper, 6 hour excerpt, recording starts at 11:00:00, the last few hours of the day interval and the beginning of the night interval are shown which are separated by a vertical line), and of the record s20101 from the sporadic group (lower, 6 hour excerpt, recording starts at 9:33:00, the last few hours of the day interval and the beginning of the night interval are shown). (For the legend see caption to figure 1.)

the LF and HF powers we used the adaptive autoregressive method with a recursive least-square algorithm [4]. The order, $M$, of the model used was 12 , while the forgetting factor, $\lambda$, was 0.985 . To assess the significant differences between different groups and intervals of records we used the Student's $t$-test.

\section{Results}

Table 1 shows number of ischemic episodes and percentages of ischemia duration per group throughout the intervals of records. The incidence of ischemia is the high- est during the morning interval for all groups. During the night interval (no physical activity) only two episodes appear in the sporadic group. In table 2 aggregate aver-

Table 1. Number of episodes per group for different intervals of records. Percentages of ischemia duration are bracketed.

\begin{tabular}{lrrrrlllr}
\hline & \multicolumn{2}{l}{ Morning } & \multicolumn{2}{c}{ Day } & \multicolumn{3}{l}{ Night } & \multicolumn{2}{c}{ Entire record } \\
\hline Salvo & 18 & $(9 \%)$ & 104 & $(6 \%)$ & 60 & $(6 \%)$ & 182 & $(6 \%)$ \\
Periodic & 27 & $(34 \%)$ & 195 & $(17 \%)$ & 60 & $(8 \%)$ & 282 & $(15 \%)$ \\
Sporadic & 23 & $(13 \%)$ & 136 & $(8 \%)$ & 2 & $(0 \%)$ & 161 & $(6 \%)$ \\
\hline
\end{tabular}




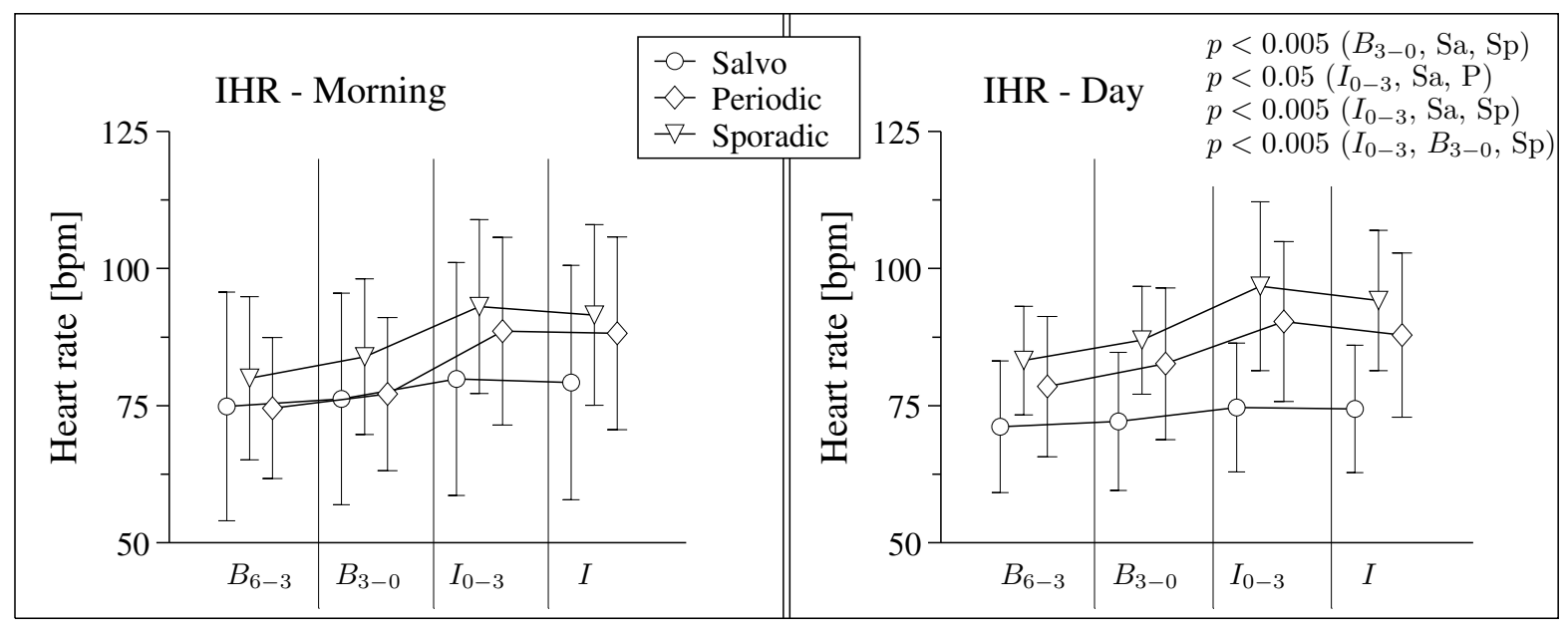

Figure 3. Aggregate average IHRs for the groups during the morning and day interval, before and during ischemia. Note that vertical bars indicate standard deviations within the intervals: $B_{6-3}$ (from 6 minutes to 3 minutes prior to ischemia), $B_{3-0}$ (from 3 minutes prior to ischemia to ischemia onset), $I_{0-3}$ (first 3 minutes of ischemia) and $I$ (during ischemia).

Table 2. Aggregate average IHRs for all groups during the intervals of records. Bracketed are standard deviations. $\dagger p<0.05$ (Morning, Night, Salvo). $\ddagger_{1} p<0.005$ (Morning, Night, Periodic). $\ddagger_{2} p<0.005$ (Day, Night, Periodic). $\ddagger_{3} p<0.05$ (Night, Entire record, Periodic). $\star_{1} p<0.001$ (Morning, Night, Sporadic). $\star_{2} p<0.05$ (Morning, Entire record, Sporadic). $\star_{3} p<0.001$ (Day, Night, Sporadic). $\star_{4} p<0.001$ (Night, Entire record, Sporadic).

\begin{tabular}{|c|c|c|c|c|}
\hline & Morning & Day & Night & Entire record \\
\hline Salvo & $74.3^{\dagger} \quad$ (13) & 69.7 & $59.1 \quad(10)$ & $66.2(11)$ \\
\hline Periodic & $78.2^{\ddagger} 1$ & $79.1^{\ddagger_{2}}(11)$ & $66.0^{\ddagger 3}$ & 74.7 (9) \\
\hline Sporadic & $79.3_{\star_{2}}^{\star 1}(11)$ & $76.9^{\star 3}$ & $62.7^{\star} 4$ & $72.4 \quad(7)$ \\
\hline
\end{tabular}

Table 3. Aggregate average ratios of the mean IHR of the intervals $I_{0-3}$ and $B_{3-0}$.

\begin{tabular}{lllll}
\hline & Morning & Day & Night & Entire record \\
\hline Salvo & 1.054 & 1.041 & 1.048 & 1.048 \\
Periodic & 1.156 & 1.096 & 1.178 & 1.139 \\
Sporadic & 1.122 & 1.117 & 1.264 & 1.126 \\
\hline
\end{tabular}

age IHRs for all groups during the intervals of records are shown. The IHR in the intervals of records is the lowest for the salvo group. For all three groups, the IHR during the morning interval significantly differs from that during the night interval. Table 3 shows aggregate average ratios of the mean IHR of the intervals $I_{0-3}$ and $B_{3-0}$. This ratio changes only slightly over the intervals of records for the salvo group. Figure 3 shows aggregate average IHRs for the groups during the morning and day interval, before and during ischemia. In the morning and day interval, for all groups, the IHR during the intervals $B_{3-0}$ and $I_{0-3}$ rises, but the increase is the least prominent for the salvo group. In the day interval, during the intervals $B_{3-0}$ and $I_{0-3}$, the
IHR for the salvo group significantly differs from that of the sporadic group.

In figure 4 aggregate average normalized $\mathrm{LF}$ and $\mathrm{HF}$ powers during the morning and day interval for all groups, before and during ischemia are shown. Generally the highest LF and HF powers are exhibited for the salvo group during the morning and day interval, while the lowest LF and HF powers are exhibited for the sporadic group in the same two intervals of records. The LF and HF powers during the interval $I_{0-3}$ (for the morning and day interval) for the salvo group significantly differ from those of the sporadic group.

\section{Discussion and conclusions}

Results in our study support the hypothesis that the salvo patterns occur due to vasospasms, and that the sporadic patterns are result of physical exertion. According to the statistics, we may conclude, that the salvo group exhibits lower heart rates, and higher sympathetic (LF power) and vagal (HF power) activity, compared to the sporadic group. Also a decrease in sympathetic activity occurs during the episodes in the sporadic, but not in the salvo group.

We might explain the patophysiology of the salvo group, where ischemia is preceded by vasospasms or by mental stress as follows: during the post-excitation phase, blood pressure may drop abruptly, and recovery of heart rate and contractility may be delayed. We may further speculate, that periodic platelet adhesion and break-off may account for recurrent vasospasms. A recent study suggests that vagal withdrawal precedes the onset of mental stress-induced ischemia [5]. Our study supports this findings.

We may describe patophysiology of the sporadic group as: ischemia is usually preceded by increase in heart rate due to physical exertion and slight increase (morning inter- 


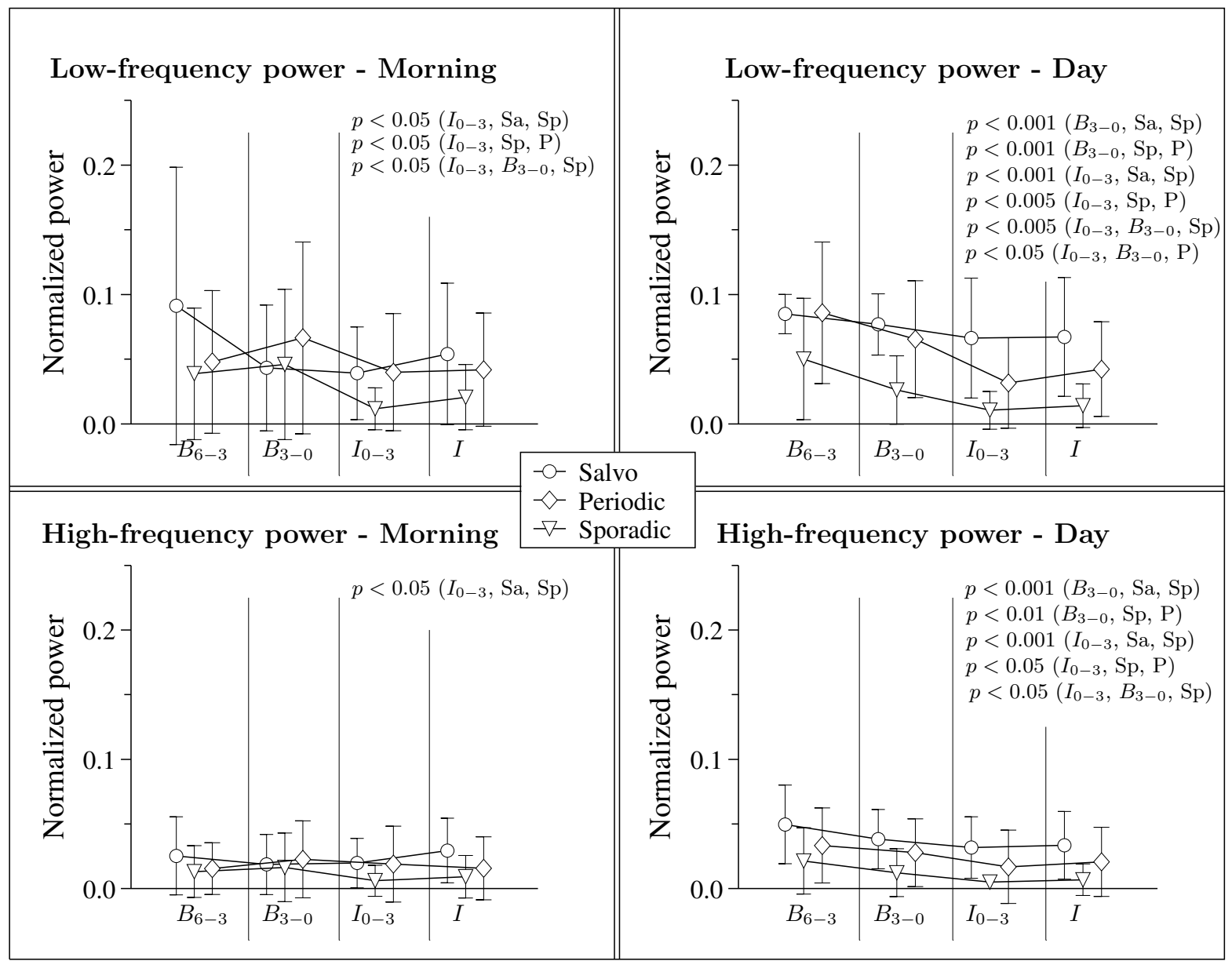

Figure 4. Aggregate average normalized LF and HF powers during the morning and day interval for all groups, before and during ischemia. Vertical bars indicate standard deviations within the intervals prior to and during ischemia (for legend see caption to figure 3 ).

val) in sympathetic activity. In this case, we would expect increased contractility, blood pressure, metabolic demand and ischemia, instead of local coronary vasodilation and increased coronary flow. Ischemia also activates complex pressor and depressor reflexes, which may alter sympathetic and vagal inputs to the cardiovascular control system. The sympatho-vagal behavior in the sporadic group is consistent with the patophysiology of effort angina.

In this study we assumed that only neurogenic factors affect coronary regulation. In addition, local endothelial factors regulate and modify blood flow in acute spastic angina, which may result in modified response. Thus, it may be inappropriate to describe the patophysiology of the salvo group in terms of sympatho-vagal influence alone.

\section{References}

[1] Jager F, Moody GB, Mark RG. Characterization of transient ischemic and non-ischemic ST segment changes. In Computers in Cardiology 1995. Los Alamitos; IEEE Computer Society Press, 1995: 721-724.
[2] Taddei A, Distante G, Emdin M, Pisani P, Moody GB, Zeelenberg C, Marchesi C. The European ST-T database: standard for evaluating systems for the analysis of ST-T changes in ambulatory electrocardiography. Eur Heart J 1992; 13: 1164-1172.

[3] Jager F, Taddei A, Moody GB, Emdin M, Antolič G, Dorn R, Smrdel A, Marchesi C, Mark RG. Long-term ST database: a reference for the development and evaluation of automated ischaemia detectors and for the study of the dynamics of myocardial ischaemia. Med Biol Eng Comput 2003; 41: 172-182.

[4] Akay M. Biomedical signal processing. Academic Press Inc., 1994.

[5] Kop WJ, Verdino RJ, Gottdiener JS, O'Leary ST, Bairey Merz CN, Krantz DS. Changes in heart rate and heart rate variability before ambulatory ischemic events. J Am Coll Cardiol 2001; 38: 742-749.

Address for correspondence:

Aleš Smrdel

University of Ljubljana, Fac. of Comp. and Inf. Sci.

Tržaška 25, 1000 Ljubljana, Slovenia

Ales.Smrdel@fri.uni-lj.si 